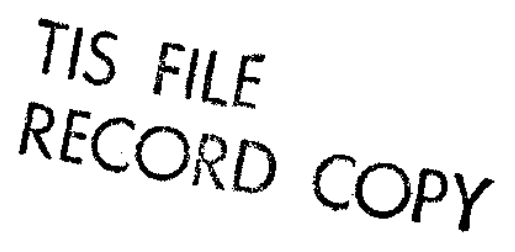

\title{
CONFIDENCE LIMITS FOR PARAMETERS OF POISSON AND BINOMIAL DISTRIBUTIONS
}

\author{
L. M. ARNETT
}

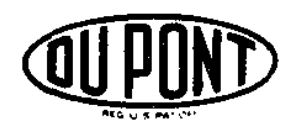

\section{SAVANNAH RIVER LABORATORY AIKEN, SOUTH CAROLINA 29801}




\section{NOTICE}

This report was prepared as an account of work sponsored by the United States Government. Neither the United States nor the United States Energy Research and Development Administration, nor any of their contractors, subcontractors, or their employees, makes any warranty, express or implied, or assumes any legal liability or responsibility for the accuracy, completeness or usefulness of any information, apparatus, product or process disclosed, or represents that its use would not infringe privately owned rights.

Printed in the United States of America

Available from

National Technical Information Service

U. S. Department of Commerce

5285 Port Royal Rood

Springfield, Virginia 22161

Price: Printed Copy \$3.50; Microfiche $\$ 2.25$ 


\section{CONFIDENCE LIMITS FOR PARAMETERS OF POISSON AND BINOMIAL DISTRIBUTIONS}

by

L. M. Arnett

Approved by

J. W. Wade, Director

Computer Sciences Section

Publication Date: April 1976

E. I. DU PONT DE NEMOURS AND COMPANY SAVANNAH RIVER LABORATORY

AIKEN, SOUTH CAROLINA 29801

PREPARED FOR THE U. S. ENERGY RESEARCH ANO DEVELOPMENT ADMINISTRATION UNDER CONTRACT AT(07-2)-1 


\section{ABSTRACT}

The confidence limits for the frequency in a Poisson process and for the proportion of successes in a binomial process have been calculated and tabulated for the situations in which the observed values of the frequency or proportion and an a priori distribution of these parameters are available. Methods are used that produce limits with exactly the stated confidence levels. The confidence interval $[a, b]$ is calculated so that $\operatorname{Pr}\{a \leqslant \lambda \leqslant b \mid c, \mu\}$, where $c$ is the observed value of the parameter, and $\mu$ is the a priori hypothesis of the distribution of this parameter. A Bayesian type analysis is used.

The intervals calculated in this report are narrower and appreciably different from results, known to be conservative, that are often used in problems of this type. Pearson and Hartley ${ }^{1}$ recognized the characteristics of their methods and contemplated that exact methods could someday be used. The calculation of the exact intervals require involved numerical analyses readily implemented only on digital computers not available to Pearson and Hartley.

A Monte Carlo experiment was conducted to verify a selected interval from those calculated. This numerical experiment confirmed the results of the analytical methods and the prediction of Pearson and Hartley that their published tables give conservative results. 
CONTENTS

\author{
Introduction 4 \\ Nature of the Problem 5 \\ Bayes' Theorem 7 \\ Poisson Processes 8 \\ One-Sided Confidence Limits 8 \\ Two-Sided Confidence Limits 11 \\ Binomial Proportion 13 \\ Monte Carlo Experiment 17 \\ References $\quad 19$
}


CONFIDENCE LIMITS FOR PARAMETERS OF POISSON AND BINOMIAL DISTRIBUTIONS

\section{INTRODUCTION}

When the frequencies of events and the proportions of successes are estimated from observational data, there are at least two methods for calculating the confidence limits for the frequency. Pearson and Hartley" have published tables "Confidence Limits for the Expectation of a Poisson Variable" and "Chart Providing Confidence Limits for $p$ in Binomial Sampling." The following comment is made on this method of estimating the confidence limits in a Poisson process: "... it is clear that in repeated sampling, particularly when $m$ (the number of times that the event occurs) is small, the proportion of occasions on which the interval includes the true expectation may be considerably in excess of the lower bound $1-2 \alpha$. Thus it should be possible to find alternative methods of solution which would fix the confidence coefficient at exactly $1-2 \alpha$ and at the same time narrow the interval." The method in this report does exactly what Pearson and Hartley contemplate, and their prediction that the exact calculation would narrow the confidence interval has indeed proven to be correct. Pearson and Hartley did what was possible with desk calculators available at the time they constructed their tables. The results of this report are only easily attainable when high-speed digital computers are used. There are many instances throughout the statistical literature. where the Pearson and Hartley tables are reproduced without the explanatory text, thereby creating an impression of exactness that the original authors apparently never intended.

What Pearson and Hartley show in their tables is

$$
\operatorname{Pr}\{c \leqslant n \mid \lambda=a\}
$$

and

$$
\operatorname{Pr}\{c \geqslant n \mid \lambda=b\}
$$

That is, the tables show the probabilities that the observed count, $c$, is as indicated given that the frequency, $\lambda$, is a fixed value. The probabilities given by these statements are easy to calculate, but they do not correspond to the situation of interest when the frequency is to be estimated from an observation. 
What is needed is

$\operatorname{Pr}\{a \leqslant \lambda \leqslant b \mid c=n\}$

That is, the count is given and what is needed is the probability that the frequency, $\lambda$, lies in the interval $[a, b]$. When the count, $c$, is sma11, the difference between the two methods may be quite large while they approach the same limit as the count increases.

Also, it is desirable to include in the calculation of the confidence limits all a priori knowledge about the process. For example, if a given pump is known to be designed by experienced engineers and manufactured by an old-line company, it is reasonable to assume a smaller frequency of failures than if it were obtained from a completely unknown source. The inclusion of this a priori knowledge is provided for in the present calculational method.

\section{NATURE OF THE PROBLEM}

Sometimes an inappropriate line of reasoning or logic will produce approximately correct results. This is the history of much of the material found in the statistical literature on the subject of confidence limits for the frequency in a Poisson process and the proportion of successes in a binomial process. Pearson and Hartley ${ }^{1}$ were apparently aware of exactly what they had calculated and assembled into Tables 40 and 41 : "... it is clear that in repeated sampling, particularly when $m$ (the number of times that the event occurs) is small, the proportion of occasions on which the interval includes the true expectation may be considerably in excess of the lower bound 1-2a. Thus it should be possible to find alternative methods of solution which would fix the confidence coefficient at exactly 1-2 $\alpha$ and at the same time narrow the interval."

Unfortunately many later authors have neglected to read or at least to note this textual material and have lifted the tables with their false appearance of exactness and have then perpetrated an incorrect line of reasoning that was never intended by Pearson and Hartley. The use of the Pearson and Hartley tables is not to be severely condemned on the basis of the numerical values because they are nearly correct and are always conservative in that the confidence level is always higher than indicated. However, the blind acceptance of the value of such tables may encourage the use of improper lines of reasoning and logic in cases where the results may be very inadequate.

The discovery and implementation of the methods, contemplated by Pearson and Hartley, to calculate the exact intervals requires 
procedures in numerical analysis that can be readily carried out only by the use of high speed digital computers. It now seems reasonable to assume that Pearson and Hartley did what was easy to do and to use desk calculators to obtain their values. Now that the exact methods are available, it seems prudent to employ the appropriate logic and to use the resultant numerical values.

The real life situation that is normally encountered and for which an appropriate analysis is to be made is typified by the following example. A process, such as the operation of motorpump combinations, has been observed for a lengthy period of time. The number of occasions on which the shaft between the motor and the pump has failed is very small, maybe zero. What value is to be used for the frequency of failure of the shaft operating in this type of service? Since the observed number of failures is zero, the maximum likelihood value of the frequency is zero. However, if the shaft can be conceived of as failing, then the true value of the frequency is some finite nonzero value and not actually zero. It is not possible to discover this fixed, true value for the frequency, but it is possible to calculate an interval and an associated probability that this interval includes the true value. It seems clear from the structure of the situation that the required statement is of the form:

$$
\operatorname{Pr}\{a \leqslant \lambda \leqslant b \mid c=n, \mu\}
$$

This is read as the probability that the frequency, $\lambda$, lies in the interval [a,b] given that the observed count, $c$, of occurrences equals $n$ and that the a priori hypothesis, $\mu$, of the distribution of $\lambda$ is what is known about the frequency before any actua 1 observations are made. For example, motor-pump shafts designed by experienced engineers and produced by reliable manufacturers are almost surely to have lower frequencies of failures than shafts obtained from a completely unknown source. All of the a priori knowledge and the observed number of failures are given as the basis for the calculation of the interval [a,b]. Any other line of reasoning or logic is incorrect and can at best give only approximate results.

Pearson and Hartley restructured the problem to calculate what is easy to calculate and to give what they recognized as only approximate results. What they calculated was

$$
\operatorname{Pr}\{c \geqslant n \mid \lambda=b\} \text { and } \operatorname{Pr}\{c \leqslant n \mid \lambda=a\}
$$

They made each expression equal to $\alpha$ and thus assigned the probability of $1-2 \alpha$ to the interval $[a, b]$. 


\section{BAYES' THEOREM}

Conditional probabilities of the form of Equation 1 can always be calculated by the use of Bayes' theorem:

$$
\operatorname{Pr}\left(A_{i} \mid B\right)=\operatorname{Pr}\left(A_{i}\right) \frac{\operatorname{Pr}\left(B \mid A_{i}\right)}{\sum_{i=1}^{n} \operatorname{Pr}\left(B \mid A_{i}\right) \cdot \operatorname{Pr}\left(A_{i}\right)}
$$

This is read as the probability of $A_{i}$ given $B$ is equal to the a priori probability of $A_{i}, \operatorname{Pr}\left(A_{i}\right)$, multiplied by the probability of $B$ given $A_{i}$ and divided by the summation of $\operatorname{Pr}\left(B \mid A_{i}\right) \cdot \operatorname{Pr}\left(A_{j}\right)$ over all values of $A_{i}$. An example will illustrate the application of Bayes' theorem. There are three boxes with identical external structures. It is known that box 1 contains two gold coins, box 2 contains two silver coins, and box 3 contains one gold coin and one silver coin. A box is selected at random, one coin is removed, and found to be a gold coin.

What is the probability that the second coin in the box is gold? The following statements represent the appropriate data:

$$
\begin{array}{ll}
A_{1}=\text { box } 1 & \operatorname{Pr}\left\{A_{1}\right\}=1 / 3 \\
A_{2}=\text { box } 2 & \operatorname{Pr}\left\{A_{2}\right\}=1 / 3 \\
A_{3}=\text { box } 3 & \operatorname{Pr}\left\{A_{3}\right\}=1 / 3 \\
B=\text { first coin is gold } & \\
\operatorname{Pr}\left(B \mid A_{1}\right)=1, \operatorname{Pr}\left(B \mid A_{2}\right)=0, \operatorname{Pr}\left(B \mid A_{3}\right)=1 / 2
\end{array}
$$

The required calculation is

$$
\begin{aligned}
\operatorname{Pr}\left(A_{1} \mid B\right) & =1 / 3\left(\frac{1}{1 \times 1 / 3+0 \times 1 / 3+1 / 2 \times 1 / 3}\right) \\
& =1 / 3\left(\frac{1}{3 / 6}\right)=2 / 3
\end{aligned}
$$

In this report, Bayes' theorem is written in differential form so that density functions replace the probabilities. The expression is now

$$
g(\lambda \mid c, \mu)=f(\lambda) \frac{h(c \mid \lambda)}{\int_{0}^{\infty} h(c \mid \lambda) \cdot f(\lambda) d \lambda}
$$

Since the frequency, $\lambda$, is continuous rather than discrete, the summation becomes an integral evaluated over all possible values 
of the frequency. The $\mu$ that follows $c$ in the given quantities on the left-hand side is merely a representation that the a priori hypothesis, $f(\lambda)$, is included on the right-hand side of the expression. Probabilities are obtained from density function by integrating over the range required to produce the desired probability. Thus,

$$
\operatorname{Pr}\{\lambda<r \mid c, \mu\}=\int_{0}^{r} g(\lambda \mid c, \mu) d \lambda
$$

An example of this method is the following. If the times of the occurrence of an event are exponentially distributed as they are for radioactive decay, the density function is

$$
f(t)=\lambda e^{-\lambda t}
$$

where $\lambda$ is a constant. The probability that a given nucleus will decay before time, $T$, equals

$$
\int_{0}^{T} \lambda e^{-\lambda t} d t=1-e^{-\lambda T}
$$

If the half-iife of the nucleus is 1 day, then $\lambda=0.693$ per day, and if $1-e^{-\lambda T}=0.95, T=4.32$ days. Therefore, it is $95 \%$ probable that a given nucleus will have decayed on or before the expiration of 4.32 days.

\section{POISSON PROCESSES}

\section{One-Sided Confidence Limits}

When rare events are distributed in time, the usual and most reasonable assumption is that the number of events in a fixed time interval has a Poisson distribution and that the intervals between events have an exponential distribution. There are at least two types of situations in which the exponential distribution holds on both a theoretical and experimental basis. If equipment is run in to eliminate manufacturing defects and is replaced, repaired, or maintained before wear becomes excessive, then the failure rate is a constant and the intervals between failures are exponential. The same distribution law holds for complex systems where individual parts are replaced as soon as they fail. ${ }^{3}$

This assumption corresponds to a constant probability of the event per unit time. Thus, 


$$
h(c \mid \lambda)=\frac{\lambda^{c} e^{-\lambda}}{c !} \quad \text { for } \quad \begin{aligned}
& c=0,1,2, \ldots \\
& \lambda \geqslant 0
\end{aligned}
$$

where $\lambda$ is the true frequency or the expected number of events for the time period of the observation, and $c$ is an observed number of events. Thus the $h(c \mid \lambda)$ in Equation 4 is fixed to the form given in Equation 6 as 1 ong as the process being considered is a Poisson process.

In Equation 4, $f(\lambda)$ can be anything that is judged suitable to account for the a priori knowledge. In this report, it is convenient, as will appear later, to choose a gamma density for $f(\lambda)$ :

$$
f(\lambda)=\frac{\beta(\beta \lambda)^{k} e^{-\beta \lambda}}{\Gamma(k+1)}
$$

where $\beta$ and $k$ are constants or parameters. The inclusion of these two arbitrary constants permits many assumptions to be made about the a priori knowledge concerning the frequency, $\lambda$. For example, if $\beta=k=0$, then $\lambda$ is uniformly distributed between zero and infinity. This means that no preference is given to any value of $\lambda$ before the experimental data are obtained. If $\mathrm{k}=0$ and $\beta$ has some positive nonzero value, then $\lambda$ is distributed exponentially and values of $\lambda$ become monotonically less likely as $\lambda$ increases.

The denominator in Equation 4 becomes

$$
\int_{0}^{\infty} \frac{\beta(\beta \lambda)^{k} e^{-\beta \lambda}}{\Gamma(k+1)} \cdot \frac{\lambda^{c} e^{-\lambda}}{c !} d \lambda=\frac{\beta^{k+1}}{c ! \Gamma(k+1)} \cdot \frac{\Gamma(c+k+1)}{(1+\beta)^{c+k+1}}
$$

Equation 4 becomes

$$
g(\lambda \mid c, \beta, k)=\frac{(1+\beta)^{c+k+1}}{\Gamma(c+k+1)} \cdot \lambda^{c+k} e^{-\lambda(1+\beta)}
$$

The $\mu$ in Equation 4 has been replaced by $\beta$ and $k$ since the a priori assumption has been made specific in Equation 7 . Equation 9 is a gamma density. Although Equation 9 may not always be amenable to integration to yield solutions in terms of known functions, at least it is a closed form of a well-known function. The result in Equation 9 was achieved by the choice of a gamma density for $f(\lambda)$. If $\beta=k=c=0$, then Equation 9 can be evaluated explicitly because it becomes 


$$
g(\lambda \mid 0,0,0)=e^{-\lambda}
$$

and

$$
\operatorname{Pr}\{\lambda \leqslant r \mid 0,0,0\}=\int_{0}^{r} e^{-\lambda} d \lambda=1-e^{-r}
$$

In the general case,

$$
\int_{0}^{r} g(\lambda \mid c, \beta, k) d \lambda
$$

is the incomplete gamma function. Numerical values for the desired integral can be obtained through its relationship to the chi-square integral that is tabulated in most statistical references: ${ }^{4}$

$$
\operatorname{Pr}\{\lambda \leqslant r \mid c, \beta, k\}=\operatorname{Pr}\left\{x^{2} \leqslant 2 r(1+\beta) \mid \nu=2(c+k+1)\right\}
$$

As an example of the application of Equation 10, let $k=2, \beta=0.5$, and $c=1$. What is the upper limit on $\lambda$ for $95 \%$ confidence? The degree of freedom, $\nu=2(I+2+1)=8$ and $\operatorname{Pr}=0.95$. The value of $\chi^{2}$ for these two entries is 15.5073. Thus the desired limit is $\lambda \leqslant 15.5073 / 2 \times 1.5=5.17$. The interval $[0.0,5.17]$ will contain the true value of the frequency, $\lambda, 95 \%$ of the time when $c, \beta$, and $k$ have the assumed values. As another example, let $k=0$, $B=0$, and $c=1$. Then $v=2(1+0+1)=4$ and for $\operatorname{Pr}=0.95, \chi^{2}=$ 9.488. Thus, the desired limit is $\lambda \leqslant 9.488 / 2=4.744$.

The choice of values for $\beta$ and $k$ in Equation 7 is governed by what is presumed known about the distribution of $\lambda^{*}$. As indicated above, the choice of $\beta=0$, and $k=0$ corresponds to a uniform distribution of $\lambda$ between zero and infinity. This implies that no value of $\lambda$ is preferred over any other value. This choice will generally yield the widest or most conservative estimates of the limits on $\lambda$ when the events are infrequent. The effect of other choices for $\beta$ and $k$ is best examined by consideration of the expected value and the variance of $\lambda$ in the gamma density. The expected value and the variance are respectively

$$
E(\lambda)=\frac{k+1}{\beta} \text { and } V(\lambda)=\frac{k+1}{\beta^{2}}
$$

If the uniform distribution of $\lambda$ seems unreasonable and low values were to be given preference over high values, then $k=0$ and $\beta>0$ might be chosen. For these cases, the limit $r$ for a given value of $\mathrm{Pr}$ and $\mathrm{c}$ is always less than for $\beta=0$ by the factor $1 /(1+\beta)$. This is the respect in which the assumption of 
a uniform distribution of $\lambda$ is said to be conservative. The only instance in which the limit $r$ is larger than for the uniform distribution of $\lambda$ is for choices of $\beta$ and $k$ that make the expected value of $\lambda$ large and the variance of $\lambda$ small. However, a small variance of $\lambda$ in the distribution implies substantial a priori knowledge about $\lambda$, and a substantial amount of experimental data will be required to modify this knowledge.

\section{Two-Sided Confidence Limits}

Two-sided confidence limits $a$ and $b$ of a Poisson frequency may be obtained by evaluating Equation 9 in the following form:

$$
\operatorname{Pr}\{a \leqslant \lambda \leqslant b \mid c, \beta, k\}=\int_{a}^{b} g(\lambda \mid c, \beta, k) d \lambda
$$

Since Equation 12 is insufficient to define a unique interval, $[a, b]$, some additional condition is needed to obtain confidence limits. The often-used practice of dividing 1-Pr equally between the lower and upper tails of the integral has the merit of ease of computation, but it produces results with undesirable characteristics. The most appropriate choice is made by imposing the condition that the interval $[a, b]$ shall be a minimum. This condition assures: (1) that as $\mathrm{P} \rightarrow 0$ and the interval $\rightarrow 0$, a and. $b$ converge to $(\mathrm{c}+\mathrm{k}) /(1+\beta)$, the maximum likelihood estimator of $\lambda$, and (2) that as $c \rightarrow \infty$, the values $a$ and $b \rightarrow$ the limits given by the normal distribution approximation to the gamma distribution. Neither of these characteristics is provided by alternative methods of specifying the interval.

The required condition is met by the simultaneous solution of Equation 12 with

$$
a^{c+k} e^{-a(1+\beta)}=b^{c+k} e^{-b(1+\beta)}
$$

Equation 13 merely states that the ordinates of the integrand are equal at $a$ and $b$. Solutions of these two equations are readily obtained with the aid of a high-speed digital computer. Table 1 is a short listing of such values. The entries are $a(1+\beta), b(1+\beta)$, and $c+k+l$ rather than twice these quantities as would appear in a table of chi-square integrals.

Table 2 gives a brief comparison of Table 1 and the values of Pearson and Hartley to indicate the differences between the two sets of data. 


\section{Table 1}

Two-Sided Confidence Intervals of a Poisson Frequency

\begin{tabular}{|c|c|c|c|c|c|c|}
\hline Confidence & Level $\rightarrow$ & .90 & & .95 & & .39 \\
\hline$c+k+1$ & $\overline{a(1+\beta)}$ & $b(1+\beta)$ & $\overline{a(1+\beta)}$ & $\overline{b(1+\beta)}$ & $a(1+\beta)$ & $b(\overline{1+B})$ \\
\hline 1 & 0.000 & 2.303 & 0.000 & 2.996 & 0.000 & 4.605 \\
\hline 2 & 0.084 & 3.932 & 0.042 & 4.765 & 0.009 & 6.643 \\
\hline 3 & 0.441 & 5.479 & 0.304 & 6.401 & 0.132 & 8.451 \\
\hline 4 & 0.957 & 6.946 & 0.713 & 7.948 & 0.393 & 10.145 \\
\hline 5 & 1.509 & 8.355 & 1.207 & 9.430 & 0.749 & 11.766 \\
\hline 6 & 2.129 & 9.723 & 1.758 & 10.864 & 1.172 & 13.327 \\
\hline 7 & 2.785 & 11.059 & 2.350 & 12.262 & 1.646 & 14.842 \\
\hline 8 & 3.467 & 12.371 & 2.974 & 13.632 & 2.158 & 16.321 \\
\hline 9 & 4.171 & 13.663 & 3.623 & 14.977 & 2.702 & 17.770 \\
\hline 10 & 4.893 & 14.938 & 4.292 & 16.304 & 3.272 & 19.195 \\
\hline 11 & 5.629 & 16.199 & 4.979 & 17.613 & 3.864 & 20.598 \\
\hline 12 & 6.378 & 17.488 & 5.681 & 18.909 & 4.476 & 21.984 \\
\hline 13 & 7.138 & 18.686 & 6.395 & 20.192 & 5.104 & 23.353 \\
\hline 14 & 7.908 & 19.915 & 7.122 & 21.464 & 5.746 & 24.708 \\
\hline 15 & 8.686 & 21.135 & 7.858 & 22.726 & 6.402 & 26.050 \\
\hline 16 & 9.472 & 22.348 & 8.603 & 23.979 & 7.069 & 27.381 \\
\hline 17 & 10.264 & 23.555 & 9.357 & 25.224 & 7.747 & 28.702 \\
\hline 18 & 11.064 & 24.755 & 10.118 & 26.462 & 8.434 & 30.013 \\
\hline 19 & 11.868 & 25.949 & 10.885 & 27.693 & 9.131 & 31.315 \\
\hline 20 & 12.678 & 27.138 & 11.659 & 28.918 & 9.835 & 32.610 \\
\hline
\end{tabular}


TABLE 2

90\% Confidence Limits for a Poisson Frequency

\begin{tabular}{lccccc}
$\begin{array}{l}\text { Number of } \\
\text { Observed } \\
\text { Occurrences }\end{array}$ & \multicolumn{2}{l}{ This Report } & & Pearson & and Hartley \\
\cline { 2 - 3 } & Lower & Upper & & Lower & Upper \\
1 & 0.000 & 2.303 & & 0.00 & 3.00 \\
2 & 0.084 & 3.932 & 0.0513 & 4.74 \\
3 & 0.441 & 5.479 & 0.355 & 6.30 \\
4 & 0.957 & 6.946 & 0.818 & 7.75 \\
5 & 1.509 & 8.355 & 1.37 & 9.15 \\
10 & 2.129 & 9.723 & 1.97 & 10.51 \\
19 & 5.629 & 16.199 & 5.43 & 16.96 \\
& 12.678 & 27.138 & 12.44 & 27.88
\end{tabular}

The a priori assumption is, in all cases, a uniform distribution of the frequency between zero and infinity. Pearson and Hartley's tables do not permit any other assumption.

\section{BINOMIAL PROPORTION}

The methods outlined above can be readily applied to the calculation of confidence limits for a binomial proportion. The density functions corresponding to $h(c \mid \lambda)$ and $f(\lambda)$ in Equation 4 are now taken to be binomial and beta densities as follows:-

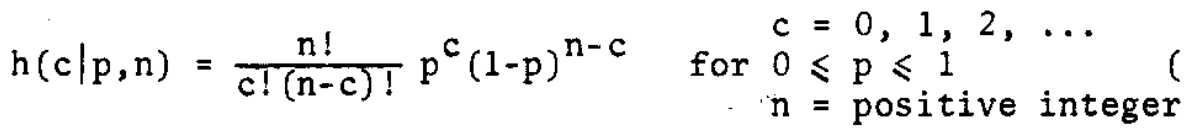

and

$$
f(p)=\frac{\Gamma(k+\ell)}{\Gamma(k) \Gamma(\ell)} p^{k-1}(l-p)^{\ell-1} \quad \text { for } \begin{aligned}
\ell & >0 \\
0 & \leqslant p \leqslant 1
\end{aligned}
$$

Then

$$
g(p \mid c, n, k, l)=\frac{\Gamma(n+k+l)}{\Gamma(k+c) \Gamma(n+l-c)} p^{c+k-1}(1-p)^{n-c+l-1}
$$

which is a beta density. 


$$
\operatorname{Pr}\{\mathrm{p} \leqslant \mathrm{r} \mid \mathrm{c}, \mathrm{n}, \mathrm{k}, l\}=\frac{\Gamma(\mathrm{n}+\mathrm{k}+\mathrm{l})}{\Gamma(\mathrm{k}+\mathrm{c}) \Gamma(\mathrm{n}+\mathrm{l}-\mathrm{c})} \int_{0}^{\mathrm{r}} \mathrm{p}^{\mathrm{c}+\mathrm{k}-1}(1-\mathrm{p})^{\mathrm{n}-\mathrm{c}+l-1} d \mathrm{p}
$$

Various assumptions concerning the a priori distribution of $p$ can be made by assigning numerical values to $\mathrm{k}$ and $\ell$. When $\mathrm{k}=\ell=1$, the assumption is that $p$ is uniformly distributed between zero and unity with no preference for any one value over another. The expected value and the variance of $p$ in the beta density are respectively,

$$
E(p)=\frac{k}{k+l} \text { and } V(p)=\frac{k l}{(k+l)^{2}(k+l+I)}
$$

Equation 17 is readily evaluated in the special case of $k=\ell=1$ and $c=0$. Then

$$
\operatorname{Pr}(p \leqslant r \mid 0, n, 1,1)=(n+1) \int_{0}^{r}(1-p)^{n} d p=1-(1-r)^{n+1}
$$

An example of the application of Equation 19 is the calculation of $r$ for $90 \%$ confidence level when five items from a lot are examined and no defects are found. Then,

$$
\begin{aligned}
0.90 & =1-(1-r)^{6} \\
r & =0.319
\end{aligned}
$$

Thus, the interval from 0.0 to 0.319 is expected to include the true proportion, $\mathrm{p}$, on $90 \%$ of the occasions.

If $k$ and $\ell$ are not unity or $c$ is not zero, the integral cannot generally be written explicitly. However, selected values are available in $F$ distribution tables. The following relationship holds: ${ }^{5}$

$$
\operatorname{Pr}\{\mathrm{p} \leqslant \mathrm{r} \mid \mathrm{c}, \mathrm{n}, \mathrm{k}, l\}=\operatorname{Pr}\left\{\mathrm{F} \leqslant\left(\frac{\mathrm{n}+\mathrm{l}-\mathrm{c}}{\mathrm{k}+\mathrm{c}}\right)\left(\frac{r}{1-r}\right) \mid \begin{array}{l}
\nu_{1}=2(\mathrm{k}+\mathrm{c}) \\
\nu_{2}=2(\mathrm{n}+\mathrm{l}-\mathrm{c})
\end{array}\right\}
$$

To find the value of $r$ in the $F$ table for the values of the previous example, the value of $F$ for $\operatorname{Pr}=0.90$ with $\nu_{1}=2$ and $v_{2}=12$ is 2.81 . Then,

$$
\begin{aligned}
2.81 & =6\left(\frac{r}{1-r}\right) \\
r & =0.319
\end{aligned}
$$

in agreement with the direct integration. 
Two-sided confidence limits for $p$ can be calculated by the same methods as described for the Poisson frequency. Equation 16 integrated between the limits of $a$ and $b$ is solved simultaneously with

$$
a^{c+k-1}(1-a)^{n-c+l-1)}=b^{c+k-1}(1-b)^{n-c+l-1}
$$

Table 3 is a short listing of two-sided confidence limits for the binomial proportion calculated by these methods.

\section{TABLE 3}

Two-Sided Confidence Limits of a Bionomial Proportion

Confidence Level $\rightarrow 0.90 \quad 0.95 \quad 0.99$

o+k Lower Limit Upper Limit Lower Limit Upper Limit Lower Limit Upper Limit

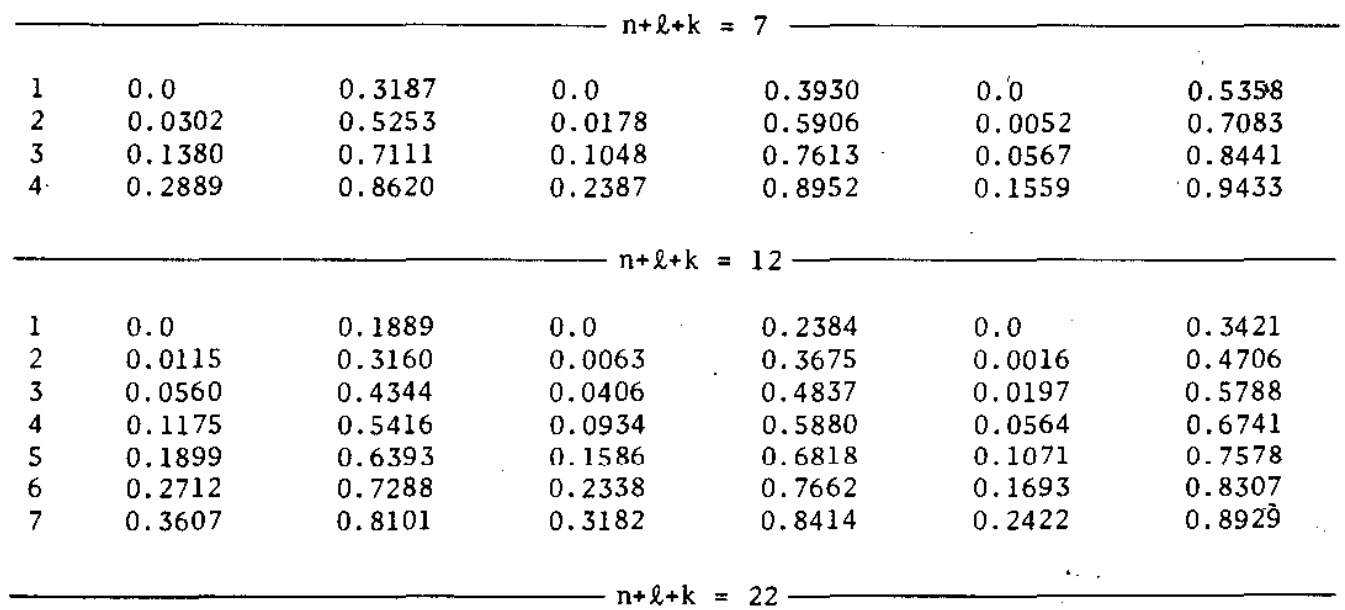

$\begin{array}{rllllll}1 & 0.0 & 0.1038 & 0.0 & 0.1329 & 0.0 \ldots & 0.1969 \\ 2 & 0.0049 & 0.1754 & 0.0026 & 0.2080 & 0.0006 & 0.2771 \\ 3 & 0.0249 & 0.2428 & 0.0176 & 0.2766 & 0.0081 & 0.3466 \\ 4 & 0.0526 & 0.3055 & 0.0409 & 0.3401 & 0.0236 & 0.4101 \\ 5 & 0.0847 & 0.3645 & 0.0692 & 0.3995 & 0.0448 & 0.4690 \\ 6 & 0.1200 & 0.4208 & 0.1012 & 0.4557 & 0.0703 & 0.5241 \\ 7 & 0.1578 & 0.4747 & 0.1361 & 0.5093 & 0.0993 & 0.5760 \\ 8 & 0.1977 & 0.5266 & 0.1734 & 0.5606 & 0.1312 & 0.6251 \\ 9 & 0.2395 & 0.5767 & 0.2129 & 0.6097 & 0.1659 & 0.6717 \\ 10 & 0.2830 & 0.6251 & 0.2544 & 0.6569 & 0.2030 & 0.7158 \\ 11 & 0.3281 & 0.6719 & 0.2978 & 0.7022 & 0.2424 & 0.7576 \\ 12 & 0.3749 & 0.7170 & 0.3431 & 0.7456 & 0.2842 & 0.7970\end{array}$


TABLE 3 (Continued)

Confidence Level $+0.90 \quad 0.95 \quad 0.99$

crk Lower Limit Upper Limit Lower Limit Upper Limit Lower Limit Upper Limit

\begin{tabular}{rllllll}
1 & & & & & \\
1 & 0.0 & 0.0716 & 0.0 & 0.0921 & 0.0 & 0.1381 \\
2 & 0.0031 & 0.1213 & 0.0016 & 0.1449 & 0.0004 & 0.1958 \\
3 & 0.0160 & 0.1683 & 0.0112 & 0.1933 & 0.0050 & 0.2463 \\
4 & 0.0338 & 0.2123 & 0.0260 & 0.2385 & 0.0148 & 0.2929 \\
5 & 0.0543 & 0.2541 & 0.0441 & 0.2812 & 0.0281 & 0.3366 \\
6 & 0.0768 & 0.2942 & 0.0644 & 0.3219 & 0.0441 & 0.3780 \\
7 & 0.1008 & 0.3330 & 0.0863 & 0.3612 & 0.0621 & 0.4176 \\
8 & 0.1260 & 0.3707 & 0.1097 & 0.3991 & 0.0818 & 0.4555 \\
9 & 0.1522 & 0.4073 & 0.1342 & 0.4359 & 0.1030 & 0.4921 \\
10 & 0.1792 & 0.4432 & 0.1597 & 0.4717 & 0.1254 & 0.5274 \\
11 & 0.2071 & 0.4782 & 0.1862 & 0.5066 & 0.1490 & 0.5616 \\
12 & 0.2357 & 0.5126 & 0.2136 & 0.5407 & 0.1737 & 0.5948 \\
13 & 0.2649 & 0.5462 & 0.2417 & 0.5740 & 0.1994 & 0.6270 \\
14 & 0.2949 & 0.5793 & 0.2706 & 0.6065 & 0.2261 & 0.6582 \\
15 & 0.3254 & 0.6116 & 0.3002 & 0.6383 & 0.2536 & 0.6885 \\
16 & 0.3566 & 0.6434 & 0.3306 & 0.6694 & 0.2821 & 0.7179 \\
17 & 0.3884 & 0.6746 & 0.3617 & 0.6998 & 0.3115 & 0.7464 \\
& & & & & & \\
\hline
\end{tabular}

$\begin{array}{llllll}0.0 & 0.0441 & 0.0 & 0.0571 & 0.0 & 0.0863 \\ 0.0018 & 0.0750 & 0.0009 & 0.0901 & 0.0002 & 0.1233 \\ 0.0093 & 0.1043 & 0.0064 & 0.1206 & 0.0029 & 0.1558 \\ 0.0196 & 0.1318 & 0.0151 & 0.1491 & 0.0085 & 0.1860 \\ 0.0316 & 0.1581 & 0.0255 & 0.1763 & 0.0161 & 0.2145 \\ 0.0447 & 0.1835 & 0.0372 & 0.2024 & 0.0252 & 0.2418 \\ 0.0585 & 0.2081 & 0.0498 & 0.2277 & 0.0354 & 0.2680 \\ 0.0730 & 0.2321 & 0.0632 & 0.2522 & 0.0466 & 0.2934 \\ 0.0880 & 0.2557 & 0.0771 & 0.2762 & 0.0585 & 0.3181 \\ 0.1034 & 0.2788 & 0.0916 & 0.2997 & 0.0710 & 0.3421 \\ 0.1193 & 0.3015 & 0.1065 & 0.3228 & 0.0842 & 0.3656 \\ 0.1355 & 0.3239 & 0.1219 & 0.3455 & 0.0978 & 0.3886 \\ 0.1519 & 0.3461 & 0.1376 & 0.3678 & 0.1119 & 0.4112 \\ 0.1687 & 0.3679 & 0.1536 & 0.3898 & 0.1264 & 0.4333 \\ 0.1857 & 0.3895 & 0.1699 & 0.4115 & 0.1413 & 0.4551 \\ 0.2030 & 0.4108 & 0.1865 & 0.4329 & 0.1566 & 0.4765 \\ 0.2205 & 0.4319 & 0.2034 & 0.4541 & 0.1722 & 0.4976 \\ 0.2382 & 0.4528 & 0.2206 & 0.4750 & 0.1881 & 0.5183 \\ 0.2561 & 0.4735 & 0.2380 & 0.4957 & 0.2044 & 0.5387 \\ 0.2743 & 0.4940 & 0.2556 & 0.5161 & 0.2210 & 0.5589 \\ 0.2926 & 0.5143 & 0.2734 & 0.5363 & 0.2378 & 0.5787 \\ 0.3111 & 0.5344 & 0.2915 & 0.5563 & 0.2549 & 0.5983 \\ 0.3298 & 0.5544 & 0.3098 & 0.5760 & 0.2723 & 0.6176 \\ 0.3486 & 0.5741 & 0.3283 & 0.5956 & 0.2900 & 0.6366 \\ 0.3677 & 0.5937 & 0.3470 & 0.6149 & 0.3080 & 0.6553 \\ 0.3869 & 0.6131 & 0.3660 & 0.6340 & 0.3262 & 0.6738 \\ 0.4063 & 0.6323 & 0.3851 & 0.6530 & 0.3447 & 0.6920\end{array}$




\section{MONTE CARLO EXPERIMENT}

Monte Carlo experiments are procedures normally performed on digital computers, wherein probabilistic processes are simulated by drawing random numbers from the appropriate distributions. The results are independent of the analytical methods that might otherwise be used to obtain a solution. Whenever a problem is complex and only one approximate answer is needed, a Monte Carlo solution may be most economical. The answer by the Monte Carlo method is never exact bccause it is always subject to random statistical fluctuations, and thus each answer obtained by using a different set of random numbers will differ slightly. A Monte Carlo calculation may also be used to provide a degree of confirmation of an analytical solution. A Monte Carlo experiment was run to confirm an entry in Table 1 . The agreement was excellent, and the results tend to confirm all of the methods used in this report, the calculated tables, and the comments by Pearson and Hartley.

A Monte Carlo experiment was conducted to examine the validity of a selected set of limits in Table 1. The limits for $c=5, \beta=0$, and $\mathrm{k}=0$ at $90 \%$ and $95 \%$ confidence were chosen to define the conditions of the experiment. The overall sample space was taken as Poisson processes whose frequency, $\lambda$, is uniformly distributed between zero and infinity. A sample process was drawn at random from this space. This particular process was observed for unit time, and if the count was 5 , the frequency was added to the final sample. If the observed count was other than 5 , the sample was ignored. The sampling from the overall population was continued until 10,000 processes, each giving an observed count of 5 , had accumulated. The distribution of frequencies was then examined. The data are shown in Table 4.

TABLE 4

Results of Monte Carlo Experiment

$\begin{array}{lc}\text { Range of Frequency } & \text { Observations } \\ 0<\lambda \leqslant 1.758 & 92 \\ 1.758<\lambda \leqslant 1.970 & 71 \\ 1.970<\lambda \leqslant 2.129 . & 58 \\ 2.129<\lambda \leqslant 9.723 & 8997 \\ 9.723<\lambda \leqslant 10.510 & 276 \\ 10.510<\lambda \leqslant 10.864 & 111 \\ 10.864<\lambda \leqslant 15.0 & 370 \\ 15.0<\lambda \leqslant 21.0 & 25 \\ 21.0<\lambda & 0 \\ 0<\lambda \leqslant 21.0 & 10,000\end{array}$


The $90 \%$ confidence limits for this experiment (Table 1) were 2.129 and 9.723 , and the $95 \%$ limits were 1.758 and 10.864 . The observed percentages of counts in these two intervals were $89.97 \%$ and $95.13 \%$, respectively, in excellent agreement with the stated confidence levels. The $90 \%$ limits of 1.97 and 10.51 from Pearson and Hartley ${ }^{1}$ included $93.31 \%$ of the observations in agreement with their warning that the listed interval would include the true frequency more often than the stated confidence.

This experiment was conducted on a digital computer by the following procedure. A random number, $n_{1}$, with a uniform distribution from 0 to 25 was generated. This random number, $n_{1}$, was taken as the true frequency, $\lambda$, of the Poisson process. The probability of a count of 5 was calculated as

$$
\operatorname{Pr}\left(5 \mid n_{1}\right)=\frac{n_{1} 5 e^{-n_{1}}}{120}
$$

A second random number, $n_{2}$, with a uniform distribution between zero and unity was generated. If $n_{2} \leqslant \operatorname{Pr}\left(5 \mid n_{1}\right)$, then $n_{1}$ was added to the sample of frequencies. The process continued until 10,000 frequencies accumulated in the sample. 


\section{REFERENCES}

1. E. S. Pearson and H. O. Hartley (Editors). Biometrika Tables for Statisticians. Vol. I, University Press, Cambridge, England (1962).

2. A. Papoulis. Probability, Random Variables, and Stochastic Processes. Chapter 4, McGraw-Hill Book Co., New York (1965).

3. R. E. Barlow and F. Proschan. Mathematical Theory of Reliability. John Wiley and Sons, Inc., New York (1965).

4. M. Abramowitz and I. A. Stegun (Editors). Handbook of Mathematical Functions with Formulas, Graphs, and Mathematical Tables. U. S. National Bureau of Standards, Applied Mathematics Series 55, U. S: Government Printing Office, Washington, D. C. (Ninth Printing, 1970) (Formula 26.4.19).

5. Ibid. (Formula 26.5.28).

6. G. J. Hahn and S. S. Shapiro. Statistical Models in Engineering. John Wiley and Sons, Inc., New York (1968). 